\title{
La I Guerra Mundial en la retaguardia: la mujer protagonista
}

\author{
Graciela Padilla Castillo \\ Universidad Complutense de Madrid \\ gracielapadilla@ccinf.ucm.es \\ Javier RoDRÍGUEz TORRES \\ Universidad de Castilla-La Mancha \\ javier.rtorres@uclm.es
}

Recibido: 3 de junio de 2013

Aceptado: 2 de julio de 2013

\section{Resumen}

Este estudio pretende revivir una parte obviada y silenciada de la historia de la Primera Guerra Mundial: el papel de la mujer. Para ello, se han consultado fuentes especializadas que hablaran de las mujeres en la línea de guerra y en la retaguardia, en los puestos de trabajo que abandonaron forzosamente los hombres para ir a combatir. El conflicto bélico paralizó algunos adelantos conseguidos hasta entonces, sobre todo en materia de sufragio universal. Sin embargo, las consecuencias sociales y políticas de la guerra también modificaron los estereotipos tradicionales de género y dieron paso a una nueva mujer moderna, que no se limitaba a vivir en el ámbito privado del hogar.

Palabras clave: I Guerra Mundial; mujer; estereotipos; historia de las mujeres.

\section{The First World War in the rear: The protagonism of Women}

\begin{abstract}
This study aims to revive an overlooked and silenced part of the history of World War I: the role of women. To this end, specialized sources have been consulted to speak of women in war line and in the rear, in jobs that men left necessarily to go fight. The war halted some progress made so far, especially in terms of universal suffrage. However, the social and political consequences of the war also changed the traditional gender stereotypes and gave way to a new modern woman, which was not confined to living in the private sphere of home.
\end{abstract}

Key words: World War I; women; stereotypes; women's history.

\section{Referencia normalizada}

Padilla Castillo, G. y Rodríguez Torres, J. (2013) La I Guerra Mundial en la retaguardia: la mujer protagonista. Historia y Comunicación Social. Vol. 18, págs. 191-206.

Sumario: 1. Introducción. 2. Estado de la cuestión y objetivo de la investigación. 3. Metodología. 4. Invisibilidad de la mujer en la guerra. 5 . El comienzo de la guerra: paralización de un incipiente proceso. 6. El impacto de la guerra en las mujeres. 6.1. Aspecto social. 6.2. Aspecto político. 7. Consecuencia: inicio de la trasformación del estereotipo femenino. 8. Conclusiones. 9. Bibliografía. 


\section{Introducción}

Para comenzar este retrato sobre la mujer protagonista en la retaguardia, durante la I Guerra Mundial, creemos necesario, en primer lugar, rescatar una cita de Marcuse (2010): "Lo ausente debe hacerse presente porque la mayor parte de la verdad reside en lo que está ausente". Entendemos que es buen resumen de la historia de las mujeres en la Primera Gran Guerra y del espíritu que quiere transmitir el presente estudio. La razón principal es que las monografías históricas y biográficas suelen hacer una historia del hombre y no una historia de las mujeres o del ser humano. El episodio bélico al que nos referimos no es una excepción. La mayoría de los tratados ha obviado la figura y la tarea de la mujer, que quedó en la retaguardia pero ni mucho menos, en segundo plano.

Berger (1975: 13) exponía una idea similar sobre los peligros que tiene no hablar o no reconocer algunos hechos:

Lo que sabemos o lo que creemos afecta al modo en que vemos las cosas. En la Edad Media, cuando los hombres creían en la existencia física del Infierno, la vista del fuego significaba seguramente algo muy distinto de lo que significa hoy. No obstante, su idea del Infierno debía mucho a la visión del fuego que consume y las cenizas que permanecen, así como su experiencia de las dolorosas quemaduras.

Atendiendo a sus palabras, vemos la Primera Guerra Mundial de forma insuficiente e inacabada. Le falta una parte importante para ser comprendida en profundidad. La pieza de la historia de las mujeres, en este caso. Y del mismo modo, la historia de las mujeres españolas. España no participó directamente en la Gran Guerra. Nunca figura en los textos como país de uno u otro bando. No obstante, se involucró ideológicamente y algunos logros para la mujer también repercutieron al país hasta la dictadura de Franco. El mismo autor, Berger, exponía al respecto: "La vista es la que establece nuestro lugar en el mundo circundante; explicamos ese mundo con palabras, pero las palabras nunca pueden anular el hecho de que estamos rodeados por él" (Berger, 1975: 13). Detectamos que las palabras escritas sobre el conflicto bélico obviaban una parte protagonista y buscamos aquellos autores que se salieron de la norma y sí las atendieron. La vista de los historiadores tiene el peligro de ser limitada y reduccionista. Consciente o inconscientemente, puede faltar una parte que hay que contar o no será recordada. ¿Por qué no miraron la tarea de la mujer en la retaguardia? Buscar las respuestas a esta pregunta requeriría otra investigación completa. Por el momento, sólo nos preocupa buscar autores que sí miraran la historia de la I Guerra Mundial de otra forma.

Para comprenderlo, recurrimos de nuevo a Berger (1975: 14): "Solamente vemos aquello que miramos. Y mirar es un acto voluntario, como resultado del cual, lo que vemos queda a nuestro alcance, aunque no necesariamente al alcance de nuestro brazo". Más adelante, expone: "Nunca miramos sólo una cosa; siempre miramos la relación entre las cosas y nosotros mismos" (Berger, 1975: 14). Sus inteligentes palabras se pueden aplicar a la historia de las mujeres: "La significación de una imagen 
cambia en función de lo que uno ve a su lado o inmediatamente después" (Berger, 1975: 37). No se queda aquí y revela:

Todo lo anterior puede resumirse diciendo: los hombres actúan y las mujeres aparecen. Los hombres miran a las mujeres. Las mujeres se contemplan a sí mismas mientras son miradas. Esto determina no sólo la mayoría de las relaciones entre hombre y mujeres sino también la relación de las mujeres consigo mismas. El supervisor que lleva la mujer dentro de sí es masculino: la supervisada es femenina. De este modo se convierte a sí misma en un objeto, y particularmente en un objeto visual, en una visión (Berger, 1975: 55).

Para conseguir lo contrario, en las líneas siguientes, buscaremos el papel protagonista de la mujer en la Primera Gran Guerra. Esto se hará desmontando su supuesta invisibilidad, explicando sus labores en la retaguardia e incluso, en el frente bélico, y buscando y comentando las consecuencias sociales y políticas del conflicto para ellas.

\section{Estado de la cuestión y objetivo de la investigación}

El apartado anterior ya remarcaba que la historia escrita y contada suele ser una historia del hombre y no de la mujer o del ser humano. Sin embargo, hemos acudido a las fuentes excepcionales que se oponen a esta norma y que sí permiten conocer el protagonismo de las mujeres en la I Guerra Mundial. No son monografías dedicadas a la mujer en la I Guerra Mundial, pero sí tratan su perspectiva en algún capítulo (Renouvin, 1948 y 1972; Perre, 1962; Fuller, 1963). También podemos destacar la monografía de Sullerot (1968) sobre la historia y la sociología del trabajo femenino, la obra de Mulvey y Richards (1998) acerca de los cambios de las mujeres entre 1890 y 1910, y el trabajo de Bordo (2004) sobre la evolución del feminismo, la cultura occidental y el cuerpo.

Para comprender la escasez de fuentes es suficiente acudir a rigurosas bases de datos científicas. Por ejemplo, Dialnet, de la Universidad de La Rioja, que reúne documentos en abierto de 58 bibliotecas universitarias, 4 bibliotecas públicas y 15 bibliotecas especializadas, sólo halla 15 entradas para las búsquedas "mujer primera guerra mundial" y "mujer primera y guerra mundial". Entre esas entradas, hay 13 documentos en castellano y 2 documentos en inglés. Siendo Dialnet una base tan fiable y democrática, la ausencia de investigaciones de este tipo entre sus documentos es representativa del vacío bibliográfico y académico generalizado sobre la mujer en la Gran Guerra. Hemos empleado todos los documentos propuestos por esta Web, además de otros documentos encontrados en nuestra carrera investigadora, que no trataban el asunto como elemento principal del título pero sí lo hacían en sus textos. Por ello, al mismo tiempo, este artículo puede ser una prospección académica para futuras investigaciones que continúen las líneas propuestas e intenten rellenar el vacío histórico. 


\section{Metodología}

Para conocer y revisar el papel de las mujeres en la I Guerra Mundial, hemos empleado una revisión bibliográfica, en versión analítica-cualitativa. Además de Dialnet, hemos buscado investigaciones especializadas, publicaciones sobre historia y género, diferentes teorías, escritos de autores y autoras que trataran el asunto, y legislación sobre la época. Esos textos son fuentes primarias especializadas: artículos, libros, legislación y estadísticas. Posteriormente, se ha analizado de forma exhaustiva toda la documentación, intentando unir enfoques que cubriesen todas las partes implicadas.

Sotelo (2012: 220) expone así las virtudes de la revisión bibliográfica y documental: "Constituye uno de los principales pilares en los que se sustenta toda investigación". El mismo autor argumenta que dicha revisión facilita la elaboración del marco teórico y permite delimitar con mayor precisión el objeto de estudio y constatar el estado de la cuestión (Sotelo, 2012: 220). Por otro lado, Charlot (1994: 1) afirma que "una buena metodología es una metodología que permite recoger, de manera rigurosa, datos pertinentes para responder a un conjunto de preguntas". En este caso, todas nuestras preguntas se referían a conocer en profundidad la situación, pública y privada, de la mujer en la Primera Gran Guerra.

Del mismo modo, queremos recordar que una revisión de metodología cualitativa, como indica su propio nombre, siempre debe tener como objetivo la descripción de las cualidades de un fenómeno. Aquí hemos buscado todo lo que rodeaba la realidad de la mujer en la I Guerra Mundial. No se trata de probar en qué grado una cierta cualidad se encuentra en ese acontecimiento, sino de descubrir tantas cualidades y orientaciones como sea posible. Por ello, buscamos el entendimiento en profundidad, en lugar de la exactitud de una investigación meramente cuantitativa.

\section{Invisibilidad de la mujer en la guerra}

Aun sabiendo de su presencia desde el comienzo de la historia de la humanidad, en los estudios historiográficos existentes, las mujeres no han sido consideradas como sujetos históricos. Estas omisiones, sin duda, se deben a una arraigada y androcéntrica concepción de la historiografía y por tanto, del objeto de esa misma historiografía. La mujer ha sido invisibilizada, ocultada y silenciada. Respecto a esta invisibilidad, Joan Scott (1992: 38-39) argumentaba:

La invisibilidad de las mujeres [...] se debe a que la ideología de esferas separadas ha definido a las mujeres como seres exclusivamente 'privados', negando así su capacidad de participar en la vida pública, política [pero] las investigaciones recientes han mostrado no el que las mujeres fuesen inactivas o estuviesen ausentes en los acontecimientos históricos, sino que fueron sistemáticamente omitidas en los registros oficiales. 
Del mismo modo, el papel de la mujer ha sido empequeñecido en los distintos contextos y procesos sociales, a pesar de su importancia. Camarero y Marcos (2012: 18) explican, precisamente, la importancia que tiene el citar siempre "el grado de desarrollo de cada sociedad, el rol alcanzado por la mujer respecto al hombre, los planes educativos y la fluctuación de este fenómeno". Caben algunas excepciones muy aisladas y descontextualizadas históricamente, con trabajos de orientación tradicional, positivista y liberal, que se centraron en "mujeres notables", enfatizando las biografías consideradas excepcionales o por su relación con y al lado de un hombre relevante: Anne Hathaway y William Shakespeare, Anna Magdalena Wilcke y Johann Sebastian Bach, Constanze Weber y Wolfgang Amadeus Mozart, Mary Ann Todd y Abraham Lincoln, Pierre Curie y Marie Curie, Martha Bernays y Sigmund Freud, Kasturba Gandhi y Mahatma Gandhi, Mileva Maric y Albert Einstein, Alma Reville y Alfred Hitchcock, Pilar Belzunce y Eduardo Chillida...

El redescubrimiento y la reconstrucción del papel de la mujer, es decir, su inclusión en la complejidad de las relaciones de contexto y procesos sociales, tiene que suponer la superación de estudios de "mujeres notables" y al mismo tiempo, la compresión global de género, incorporando a distintos estudios históricos la orientación hacia la vida privada, emociones y actitudes de la mujer. Siguiendo a Mary Nash (2004), hay que releer la historia con perspectiva de género y no sólo rescatar el protagonismo de las mujeres en el pasado, sino presentar instrumentos para repensar la dinámica histórica en su conjunto, el papel decisivo del ámbito privado en el desarrollo histórico y el papel de las mujeres en ese ámbito.

La guerra, como contexto y proceso social, no constituye una excepción. El vocablo procede de un grito de combate de origen germánico, "werra". De este grito surgen los actuales "wehr" alemán, "war" inglés, "guerre" francés y "guerra" en español. Más bien al contrario, en el escenario de la guerra, se incrementa y amplía la exclusión de la mujer. Su papel quedó al margen: sin nombre, sin perfil, sin voz, sin territorio, sin actuación. Las bases ideológicas de género que han sustentado este discurso se asentaban en el ideario de la domesticidad: el culto de la maternidad y la familia como máximo horizonte de realización femenina. La representación más frecuente de la mujer es denominada, como mencionan las autoras Radl Philipp y García Negro (1993), "el Ángel del Hogar".

Sin embargo, esta expresión no es, en absoluto, una invención de los años noventa del pasado siglo. Desde que aparecen las primeras sociedades, incluso en muchos territorios de la actualidad, las actividades bélicas se identificaban con lo masculino. Se consideraba que el varón tenía mayor inteligencia, capacidad para el autocontrol, más fuerza física y mental. El hombre mandaba y la mujer obedecía, dentro y fuera del hogar.

Por otro lado, es interesante estudiar esa invisibilidad en la guerra, porque la guerra ha sido, a lo largo de la historia, la relación más importante entre los pueblos. Tomando como referencia los datos del Instituto Henry Dunant (Pictet, 1986: 93), 
durante 5.000 años de historia, se han producido 14.000 guerras, que han causado la muerte de 5.000 millones de seres humanos.

Asimismo, el desarrollo y progreso del ser humano han afinado las diferentes formas de civilización. El perfeccionamiento del aparato militar trae como consecuencia directa el aumento de víctimas, tanto militares como de la población civil. La mujer, como parte de la población civil, y obviada en cualquier toma de decisión, resulta ser, sobre todo, víctima y por lo general, son ellas las que calladamente, en periodos de guerra, han garantizado la supervivencia de su familia e incluso, de su comunidad.

Hasta el siglo XX, la participación de las mujeres en la guerra fue excepcional e invisible. Será a partir de la Primera Guerra Mundial cuando las mujeres inicien su participación de una forma más perceptible. Esta guerra movilizó enormes ejércitos: unos 65 millones de soldados entre todos los contendientes. Provocó que la retaguardia quedara huérfana de mano de obra y hubo que recurrir a la mujer para mantener la producción. A modo de ejemplo, 430.000 mujeres francesas y 800.000 mujeres británicas pasaron de ser amas de casa a obreras asalariadas e incluso, muchas de ellas forman parte de la propia guerra. En Alemania, sin participar directamente en las unidades de combate, contribuyeron en las actividades de la guerra, trabajando en las fábricas de armamentos y desarrollando diversas tareas cerca del frente de batalla: avituallamiento, depósito de municiones, etc. Poco antes de terminar la guerra, casi 68.000 mujeres reemplazaron a los hombres que estaban en el frente.

En Inglaterra, las mujeres también participaron en el conflicto bélico como civiles, con o sin remuneración. De hecho, 80.000 mujeres se enrolaron como auxiliares en las unidades femeninas de las fuerzas armadas. Constituyeron WAAC (Women's Auxiliary Army Corps o Cuerpo femenino Auxiliar del Ejército), WRNS (Women's Royal Naval Service o Servicio Femenino de la Real Armada) y WRAF (Women's Real Aerial Force o Real Fuerza Aérea Femenina). Al mismo tiempo, otras tantas prestaron servicio como enfermeras. Y en Rusia, aunque en menor número, también participaron en los combates. Formaron unidades de combate de mujeres voluntarias, bajo la autorización del ministro de guerra y líder revolucionario, Aleksandr Fiódorovich Kérenski. Maria Leontievna Bochkareva, más conocida por su apodo, Yashka, formó el primer batallón integrado exclusivamente por mujeres, conocido como el Batallón de la Muerte de Mujeres. Bochkareva ya había formado parte de diversas unidades de composición mixta pero en esta nueva tarea, reunió y formó a 2.000 mujeres.

España, habiendo perdido ya los últimos territorios del Imperio colonial, aparecía al margen de los asuntos continentales que ocupaban las claves diplomáticas de esta época. Era una pequeña potencia con intereses reducidos al norte de África, a la sombra de los intereses coloniales de las potencias europeas. De esta realidad que nos dibuja cualquier aproximación a la situación general de los medios militares españoles, al comenzar la Primera Gran Guerra, se deducirá la "opción” española frente a la guerra o, más bien, su necesaria neutralidad a tenor de sus grandes limitaciones mili- 
tares y otros conflictos internos. Como balance para España, la contienda contribuyó a incrementar la inestabilidad política (aliadófilos y germanófilos), más incapaz aún de hacer frente a las insuficiencias militares y a los desequilibrios económicos, con la consiguiente conflictividad social, generados por la guerra.

Por otro lado, y volviendo al resto de países, el gigantesco desarrollo de los medios de guerra, a comienzos de ese siglo, acrecentó la incidencia de muertes para la población civil -ancianos, mujeres y niños-. Además, durante la contienda, se produjeron 21 millones de muertes a causa de las enfermedades que acompañan a los conflictos armados, por el deterioro de las condiciones sanitario-higiénicas y las epidemias. De hecho, las muertes por epidemias incidieron mayoritariamente sobre la población civil.

\section{El comienzo de la guerra: paralización de un incipiente proceso}

Las líneas anteriores nos han permitido un acercamiento al papel de la mujer en la I Guerra Mundial. Sin embargo, todo estudio histórico debe empezar por los antecedentes. Así, nos preguntamos por la situación previa de la mujer antes de las hostilidades y los documentos, especialmente legislativos. Estos nos mostraron que el conflicto bélico detuvo el desarrollo del sufragio universal. Parece que el voto de la mujer quedó relegado varios planos, porque las penurias de la guerra desviaron la atención a otras perspectivas políticas, sociales y económicas.

No obstante, nos parece de vital importancia descubrir esta interrupción de los primeros pasos que ya se habían dado hacia el sufragio universal. La primera mención podemos encontrarla en la Declaración Universal de los Derechos del Hombre y del Ciudadano, de 1789. Ésta se inspiró en la Declaración de la Independencia de Estados Unidos, firmada en 1776. Desde el 14 de julio hasta el 26 de agosto, la Asamblea, constituida durante la Revolución Francesa, estudió y modificó varios textos. Tras muchos debates, la Declaración fue aprobada y ratificada por el monarca Luis XVI, el 5 de octubre. Su preámbulo manifiesta que la Asamblea Nacional se ha reunido para crear una "declaración solemne" que exponga "los derechos naturales, inalienables y sagrados del hombre". Por ello, su primer artículo expone que todos los hombres "nacen y permanecen iguales en derechos". Según el texto, las desigualdades o distinciones sociales sólo pueden provenir de la utilidad común.

Uno de esos derechos fundamentales de todos los hombres es la libertad. Según el artículo 4, el ejercicio de esa libertad sólo tiene como límite la libertad del otro. Los hombres son libres siempre que respeten la libertad de sus iguales. De hecho, el siguiente artículo dice que la Ley sólo prohibirá los actos perjudiciales y que todo lo que no esté dentro de esas prohibiciones será permitido. El objetivo es que la Ley sea la expresión de la voluntad general (artículo 6) y que todos los ciudadanos contribuyan a crearla y mantenerla, de forma personal o a través de sus representantes. El artículo 10 anuncia que nadie será incomodado por sus opiniones, incluidas las religiosas, siempre que no perturbe el orden público. Por su parte, el artículo 11 cita 
la libre comunicación de pensamientos y de opiniones y lo califica como "uno de los derechos más preciosos del hombre". Este derecho permite a todo ciudadano "hablar, escribir e imprimir libremente, a trueque de responder del abuso de esta libertad en los casos determinados por la ley". Por último, el artículo 17 habla del derecho a la propiedad, también como inviolable y sagrado. Ninguna persona puede ser privada de él salvo cuando la necesidad pública lo exija.

Muchos autores han estudiado y comentado estos artículos fundamentales desde una perspectiva de género. Entre los más recientes, se encuentran el trabajo de Martínez (2011: 58-70), que los relacionaba con el tratamiento informativo de violencia de género; los estudios de las producciones discursivas de las mujeres en Latinoamérica de Del Valle y Mejías (2011: 60-68); las competencias, conocimientos, habilidades y actitudes no discriminatorias en las aulas (Rodríguez, 2010: 245-270); o las traslaciones a heroínas actuales de ficción de Padilla (2009b: 20-35 y 2010: 173-188).

Volviendo a los artículos de la Declaración Universal de los Derechos del Hombre y del Ciudadano, todos los ciudadanos nacen y viven iguales ante la ley. Su libertad acaba donde pueden menoscabar o dañar la libertad del otro. Tienen libertad de pensamiento, expresión y religión; y derecho a la propiedad. Estas ideas, que eran tan avanzadas y supuestamente para todos, fueron traicionadas en los primeros años de la revolución, ya que no se llevaron siempre a la práctica. De hecho, la siguiente protagonista moriría guillotinada por ejercer su derecho a la libertad de expresión. Dos años después de la Declaración Universal de los Derechos del Hombre y del Ciudadano, Olimpia de Gouges denunció el texto porque no se mencionaba a las mujeres. La Declaración sólo hablaba de "hombre", "hombres", "ciudadano" y "ciudadanos". Por ello, esta escritora y pensadora política francesa decidió redactar un nuevo texto, igualitario de verdad. Este acto y su enfrentamiento con Robespierre y Marat la llevarían a la muerte, precisamente por escribir una carta pública contra el primero (Pronostic de Monsieur Robespierre pour un animal amphibie). Parecía radical para su época, aunque sólo buscaba el trato igualitario para la mujer y el hombre. Expresó sus ideas feministas en novelas, obras de teatro, textos periodísticos en el periódico que dirigió, L'Impatient, y en reuniones de la Societé Populaire de Femmes, de la que fue fundadora. Su logro principal fue, sin duda, la Declaración Universal de los Derechos de la Mujer y la Ciudadana, con fecha de 1791. Su preámbulo es claro y directo:

Las madres, las hijas, las hermanas, representantes de la Nación, solicitan ser constituidas en Asamblea Nacional. Considerando que la ignorancia, el olvido o el desprecio de los derechos de la mujer son las únicas causas de las desgracias públicas y de la corrupción de los gobiernos, han decidido exponer en una solemne declaración los derechos naturales, inalienables y sagrados de la mujer, con el fin de que esta declaración, presente continuadamente en la mente de todo el cuerpo social, les recuerde sin cesar sus derechos y deberes; con el fin de que los actos de poder de las mujeres y los actos de poder de los hombres puedan ser comprados en cualquier momento con el objetivo de toda institución política, y sean más respetados; con el fin de que las reclamaciones de las ciudadanas, basadas en lo sucesivo sobre principios sencillos e incontrovertibles, tiendan siempre hacia el mantenimiento de la Constitución, de las buenas costumbres y de la felicidad de todos. 
Olimpia de Gouges hablaba de las mujeres, que quedaron olvidadas en la Declaración Universal de los Derechos del Hombre y del Ciudadano. Su estructura es muy similar al primer texto, por lo que reproduce los mismos artículos introduciendo los cambios oportunos. Podemos encontrar las diferencias en los mismos artículos que hemos reproducido anteriormente. Por ejemplo, el primer artículo argumenta que la mujer "nace libre y permanece igual al hombre en derechos". El artículo 4, que hablaba de libertad, expone que los derechos de las mujeres han estado limitados por la tiranía perpetua del hombre. Por ello, no se ha cumplido aquello de que la libertad de la persona sólo tiene límites donde empieza la del otro. Olimpia de Gouges dice que los límites deben ser modificados "por las leyes de la naturaleza y de la razón", para que se contemplen a las mujeres y sus derechos. Por su parte, el artículo 6 vuelve a indicar que la Ley es la expresión de la voluntad general y que las mujeres también deben contribuir, pues todos los ciudadanos son iguales. El artículo 10, que hablaba de las opiniones, incluye una idea importantísima: el derecho de la mujer a subir al cadalso o hablar en público. Este derecho no se había contemplado expresamente en la Declaración Universal de los Derechos del Hombre y permite sacar a las mujeres del ámbito privado, al que han sido relegadas. Por último, el artículo 11 vuelve a incidir en la libertad de pensamiento y opinión como "uno de los derechos más valiosos de la mujer", y el artículo 17 en el derecho de propiedad, "de todos los sexos unidos o separados", algo que no se contemplaba anteriormente.

Lo sorprendente es que estas ideas serían olvidadas, sin llegar a ser concretadas hasta muchos años después, ya en el siglo XX. Como indicamos al comienzo de este apartado, la I Guerra Mundial paralizó el diálogo abierto e incluso, la Segunda Gran Guerra volvió a detener la aparición del sufragio universal. El paso decisivo no llegaría hasta 1947, cuando la Comisión sobre la Condición Jurídica y Social de la Mujer de Naciones Unidas creó un borrador que pretendía ser instrumento legal para articular los derechos de hombres y mujeres. Según indica García-Berrio Hernández (2008: 146), sus años de mayor actividad fueron los comprendidos entre 1975 y 1985, al haber dado lugar a las tres primeras Conferencias Mundiales de las Naciones Unidas sobre la mujer.

Antes de llegar a esas conferencias centradas en la mujer, hay que repasar otros hitos importantes. El primero de ellos tuvo lugar en 1950, cuando Naciones Unidas cambió su Declaración Universal de los Derechos del Hombre por Declaración Universal de los Derechos Humanos. Más adelante, en 1979, la Asamblea General promulgó la Convención para la eliminación de todas las formas de discriminación contra la mujer. Según el texto, "todos los seres humanos nacen libres e iguales en dignidad y derechos", "sin distinción alguna y, por ende, sin distinción de sexo". Hombres y mujeres tienen los mismos "derechos económicos, sociales, culturales, civiles y políticos" y es preciso eliminar la discriminación contra la mujer, "en todas sus formas y manifestaciones". Elaboraron un texto de 30 artículos y exhortaron a los Estados Partes a condenar cualquier forma de discriminación, "por todos los medios apropiados y sin dilaciones". Estos medios incluían leyes que suprimieran la trata de mujeres y la explotación de la prostitución de la mujer. Los artículos siguientes 
hablaban de la igualdad de la mujer en el ámbito de la educación, el empleo, la atención médica y planificación familiar, los medios rurales y más desfavorecidos, o el matrimonio. Sin duda, todos elementos que bien podrían haber sido detectados muchos años antes.

\section{El impacto de la guerra en las mujeres}

A comienzos del siglo XX, la vida en las ciudades de Europa mejoró paulatinamente. El suministro de agua potable, la limpieza y pavimentación de las calles, el abastecimiento de gas y electricidad, entre los avances más importantes, aliviaron la mortalidad de todos los grupos sociales. Por ejemplo, en 1890, la esperanza de vida media de las mujeres en Gran Bretaña era de 44 años; en 1910, de 52,4 años; y en 1920, de casi 60 años. La mujer urbana abordaba una vida mejor. Aunque la mujer pobre tardaría más en la consecución de las mejoras. Al mismo tiempo, la extensión de la industrialización y el desarrollo del trabajo en cadena, que sustituyó la fuerza física por la máquina, favorecieron la incorporación de la mujer al trabajo. Estas condiciones de la mejora de vida tuvieron como consecuencia directa un progresivo descenso del número de miembros de la familia.

Ante la reducción de las familias y de los índices de natalidad, los gobiernos, en especial los europeos, reaccionaron con legislaciones que tenían como finalidad favorecer la maternidad. Así, la legislación de orientación proteccionista, los sindicatos y los nuevos trabajos fueron los elementos de cambio más importantes en la vida de las mujeres urbanas de clase obrera, entre la década de 1870 y la de 1920 (Anderson y Zinsser, 1991).

\subsection{Aspectos sociales}

El trabajo como actividad natural y cotidiana para los hombres, e infrecuente para las mujeres, se modificó en este de tiempo guerra. Las mujeres fueron reclamadas en el trabajo y ocuparon su espacio en las fábricas, en puestos antes reservados a los hombres. Como indican Anderson y Zinsser (1991), los "trabajos de hombres" son desempeñados temporalmente por mujeres, sin ninguna pretensión de perpetuidad.

La lectura de diversa bibliografía reseña que la I Guerra Mundial influyó en la liberación femenina y en la transformación del rol de las mujeres en la sociedad. Maurice Bardèche, periodista y escritor francés, explicaba:

Cuando el decreto de movilización hizo desaparecer, como en una trampa, a todos los varones adultos que se podían hallar entre la Bretaña y los Urales, las mujeres se hallaron de pronto en una situación que la Historia les ofrecía por primera vez. En el vacío así creado, estas desahuciadas se hallaron de repente frente a las tareas de los varones, a los útiles de los varones, a los sillones vacíos dejados por los varones... 
Sin embargo, si se reflexiona más profundamente, la idea de que la guerra fue un instrumento emancipador de la mujer no hace más que reforzar el modelo femenino de madre-ama de casa, porque la ausencia del hombre descubre a la sociedad, en su conjunto, un rol que aúna su máxima responsabilidad en el hogar y la inserción al mundo laboral.

La continuidad del conflicto, el aumento del esfuerzo bélico conforme éste se fue prolongando y la intensificación del alistamiento masivo de soldados, hicieron necesario que los gobiernos contrataran a las mujeres para que colaborasen en la retaguardia y así, evitar el colapso industrial. La siguiente tabla, elaborada por Vidaurreta (1981), muestra y distribuye por sectores de trabajo, la presencia de las mujeres trabajadoras en los años de la Gran Guerra:

Tabla 1. Porcentajes de mujeres sobre hombres empleados por sectores.

\begin{tabular}{|c|c|c|c|c|}
\hline AÑO & Industria \% & Transporte \% & Agricultura \% & Comercio \% \\
\hline $\mathbf{1 9 1 4}$ & 26 & 2 & 9 & 27 \\
\hline $\mathbf{1 9 1 8}$ & 35 & 12 & 14 & 53 \\
\hline $\mathbf{1 9 2 0}$ & 27 & 4 & 10 & 40 \\
\hline
\end{tabular}

Resulta evidente que el conflicto armado se convirtió en un medio por el cual se eliminaron las barreras que separaban los trabajos masculinos de los femeninos. Las mujeres se convirtieron en operarias, montaron aviones, trabajaron en fábricas de municiones, en los ferrocarriles y en las minas, condujeron el metro, autobuses y camiones. Se convirtieron en la primera fuerza de la retaguardia y en el campo, su trabajo resultó fundamental para la supervivencia de todos los seres humanos. Este cambio dotó de confianza a la mujer y explica su nueva y necesaria emersión en la sociedad. El economista y escritor norteamericano, Raymond Robins, en Duby y Perrot (1993: 31) afirma: "Es la hora inaugural de la historia para las mujeres del mundo. Es la era de las mujeres".

La guerra desafió el concepto de feminidad existente. La necesidad de libertad para el cuerpo, favoreciendo los movimientos, implicó una nueva indumentaria y la implementación de pantalones y chaquetas. Por otro lado, los traumas producidos durante el conflicto y la obsesión por la muerte "vuelven al amor más ávido y más banal a la vez" (Duby y Perrot, 1993: 49). Se contribuyó al "advenimiento de la pareja moderna, centrada en una exigencia de realización individual y ya no patrimonial" (Duby y Perrot, 1993: 49).

\subsection{Aspectos políticos}

La "Segunda Revolución Industrial", iniciada hacia 1870, vino acompañada de profundos cambios políticos, económicos y sociales que estimularon el movimiento feminista a finales del siglo XIX. Las metas del movimiento feminista se relacionaron con la consecución del derecho al voto, la mejora de la educación, la capacitación 
profesional y la apertura de nuevos horizontes laborales, la equiparación de sexos en la familia como medio de evitar la subordinación de la mujer y la doble moral sexual. Entendemos doble moral en el sentido victoriano, donde se considera a la mujer como ser asexual cuyo impulso es la maternidad, análogo al impulso sexual del hombre. Este movimiento, como novedad, tuvo repercusión importante al acompañarse de una movilización colectiva en los distintos países, con logros sustanciales que se vieron ralentizados pero, al mismo tiempo, consolidados durante la Primera Gran Guerra.

Hasta este momento, en la historia de la humanidad, la mujer tenía una doble rígida separación en las esferas de participación social del hombre: en la producción y en la política. La guerra posibilitó la transición por necesidad y supervivencia, a una de las esferas de participación, como hemos venido relatando: la producción. Por otra parte, en un proceso más largo, aunque ya iniciado, las manifestaciones de una conciencia y de una política feminista se encontraron en las luchas por los derechos de la mujer, los movimientos sufragistas y el movimiento obrero. La esfera de participación política y su asimilación hicieron que la subordinación de la mujer al hombre se convirtiera en un aliado oculto y le sirviera para difundirla en su tarea de socialización de los hijos.

Así, la Primera Guerra Mundial trajo consigo los primeros conceptos modernos sobre la mujer y la sociedad: la aparición, por primera vez en la historia de Europa, de una sociedad mixta propiamente dicha. Mutación de la que sólo conocíamos el parcial y, por tanto, antecedente poco representativo de la obrera trabajando al lado de sus compañeros en las fábricas surgidas a raíz de la Revolución Industrial. La mujer pudo mantener una vida pública y privada, logrando un equilibrio que favoreció su desarrollo personal y profesional. Rompió con las costumbres de la época, provocó cambios sustanciales en las relaciones familiares y maritales, hasta cambios estéticos que siguieron impulsando su emancipación.

El mejor ejemplo de este cambio político es Gran Bretaña. Allí, las sufragistas perdieron, catorce veces, pugnas parlamentarias para conseguir el derecho al voto. Sin embargo, su empoderamiento como consecuencia de su contribución a la Gran Guerra fue decisivo para su obtención. Lo consiguieron, finalmente, en 1928. España seguiría el ejemplo con la Constitución de 1931, durante la Segunda República. Las mujeres españolas ejercieron su derecho al voto, por vez primera, en las elecciones de 1933. Esto también sustenta la idea, citada en líneas anteriores, de que el país no participó en la Gran Guerra directamente pero se vio involucrado ideológicamente, con sus consecuencias negativas y positivas, como en el caso del sufragio femenino. Sin embargo, la dictadura de Franco lo anularía de nuevo hasta 1975.

\section{Consecuencia: inicio de la trasformación del estereotipo femenino}

El culmen de los cambios sociales y políticos que vivió la mujer después la Primera Guerra incluyó una modificación de los estereotipos de mujeres. Para comprenderlo, consideramos necesario definir qué son el estereotipo y el estereotipo de género. 
Atendiendo a Bernárdez, García y González (2008: 123), el estereotipo es una serie de opiniones, actitudes, sentimientos o reacciones de los miembros de una determinada comunidad o grupo respecto a otros, y por ende, tienen un carácter homogéneo y rígido. Desde el punto de vista cognitivo, consiste en la operación mental de adjudicar ciertas características a una serie de individuos por el hecho de pertenecer a un grupo. El estereotipo es un esquema que se aplica a la interacción social, es siempre una generalización, y como tal, poco exacta. Comunicativamente, responde a un principio de economía cognitiva: gracias a un estereotipo somos capaces de procesar una gran cantidad de información con menos esfuerzo cognitivo.

Desde esta perspectiva, se entiende que son la estandarización cultural y normas sociales, absorbidos durante el proceso de socialización. Más allá de una función de simplificación, los estereotipos son sistemas de valores a partir de los cuales los individuos se caracterizan a sí mismos y a los demás. Los rasgos de estereotipación varían respecto a las culturas y épocas históricas. El conjunto de cualidades y características psicológicas y físicas que una sociedad asigna a hombres y a mujeres, constituye lo que se denominan estereotipos de género. Padilla (2009a: 262) añade: "Los roles y funciones asignados a hombres y mujeres por su sexo son bien distintos e infranqueables". Los estereotipos crean arquetipos, fijan un modelo de ser hombre y un modelo de ser mujer, validados socialmente. A partir de esa visión tópica construida, establecen un sistema desigual de relaciones entre ambos sexos y de cada uno de ellos con el mundo. Estos estereotipos se han construido en función de los intereses, capacidades y tareas, que se asocian a los roles tradicionalmente diferentes asignados a cada sexo.

Los estereotipos femeninos son menos variados que los masculinos y han evolucionado bastante menos. Los roles y funciones de las mujeres y de los varones se definen y atribuyen en función de su sexo. Muchas veces, se basan sólo en características psicofísicas diferentes: por ser de uno u otro sexo, las personas tienen una determinada forma de ser y actuar. Estas ideas confirman la importancia del cuerpo y de la apariencia externa de las mujeres. Además, se basan en la idea anticuada y sexista de que el ámbito público es para los varones y el ámbito privado, para las mujeres. Del mismo modo, las mujeres se relacionan con la infancia y la juventud, de cuya educación y cuidados se considera que es la principal responsable (Dirección General de la Mujer, 2003: 254-256). Justo en estos puntos, la Primera Gran Guerra modificó los estereotipos. La revisión histórica nos ha permitido demostrar que las mujeres hicieron las mismas tareas que los hombres, en las fábricas y en la línea de guerra. Pasaron del ámbito privado al público, y su tarea dejó de estar centrada en el hogar y la crianza de los hijos.

Atendiendo a la clasificación de Bernárdez, García y González (2008: 122-129), la Primera Guerra Mundial pasó de los estereotipos tradicionales, de amas de casa o mujeres contentas con el destino de género que las espera, a mujeres modernas y sexualmente activas, que están tan preparadas como los hombres para asumir los retos de la vida moderna. Mujeres que no tienen dificultades para compaginar sus facetas pública y privada, ni sufren conflictos por los avances producidos gracias al 
movimiento de liberación de las mujeres, aunque estos aún no las liberan totalmente de los modelos de belleza convencionales.

\section{Conclusiones}

Hemos retrocedido un siglo y desde allí, hemos revivido el comienzo de la transformación de la mujer y sus primeros pasos en la conquista de su libertad. Desde las formas físicas hasta las sociales. Desde la indumentaria hasta su psicología. El tema de género, la reivindicación de la mujer y de sus derechos, su integración dentro del ámbito de la sociedad actual, son hechos en la actualidad. Sin embargo, las situaciones de desigualdad de la mujer frente al hombre continúan siendo un problema latente.

Desde distintas publicaciones, foros y asociaciones, públicas y privadas, existen enfoques que intentan luchar contra ello desde muy diferentes visiones. No obstante, $\mathrm{y}$ con frecuencia, falta una mirada profunda que oriente de forma peculiar su educación y más importante, que posteriormente, desarrolle todos sus potenciales y posibilidades en su papel elegido dentro de su entorno habitual.

Al reflexionar sobre la historia de la mujer en el siglo XX y en un tiempo difícil como fue la Primera Guerra Mundial, pretendemos reivindicar su papel histórico en la retaguardia y en la línea de guerra. Todo ello obviado y discriminado por la historiografía, generalmente androcéntrica. Asimismo, deseamos colaborar, en la medida de nuestras posibilidades, en sacar del olvido lo que las mujeres han tolerado a lo largo de la Historia; en la Guerra y en la Paz. Corroboramos que las situaciones difíciles les han dado a las mujeres un papel relacionado directamente con la libertad.

\section{Bibliografía}

ANDERSON, B. S.; ZINSSER, J. P. (1991). Historia de las mujeres: una historia propia. Barcelona: Editorial Crítica.

BEGER, J. (1975). Modos de ver. Barcelona: Editorial Gustavo Gili.

BERNÁRDEZ, A.; GARCÍA, I.; GONZÁLEZ, S. (2008). Violencia de género en el cine español: análisis de los años 1998 a 2002 y guía didáctica. Madrid: Editorial Complutense.

BORDO, S. (2004). Unbearable Weight: Feminism, Western Culture, and the Body. Berkeley: University of California Press.

CAMARERO, E.; MARCOS, M. (2012). "Campañas en televisión contra la violencia de género del Ministerio de Sanidad, Política Social e Igualdad (2008-2011). Análisis de contenidos previo al estudio de recepción". En Revista de Comunicación Vivat Academia, $\mathrm{n}^{\mathrm{o}}$ 121, Madrid: Universidad Complutense de Madrid. p. 17-30. 
CHARLOT, B. (1994). "El enfoque cualitativo en las políticas educativas". En: Perfiles educativos, $\mathrm{n}^{\circ}$ 63, México D.F.: Instituto de Investigaciones sobre la Universidad y la Educación. p. 1-4.

DEL VALLE, M. E.; MEJÍAS, N. E. (2011). "La hora de los secretos de Eleonora Gabaldón: una aproximación al discurso de género". En Revista de Comunicación de la SEECI, $\mathrm{n}^{\circ}$ 27, Madrid: Universidad Complutense de Madrid. p. 60-68.

DIRECCIÓN GENERAL DE LA MUJER (2003). ARESTE: Arrinconando Estereotipos en los medios de comunicación y la publicidad. Madrid: Dirección General de la Mujer, Consejería de Trabajo.

DUBY, G.; PERROT, M. (1993). Historia de las mujeres. Madrid: Taurus.

FULLER, J. F. C. (1963). La conduite de la guerre. París: Payot.

GARCÍA-BERRIO, T. (2008). Medidas de protección de la mujer ante la Violencia de Género. Claves para la igualdad. Madrid: Grupo Difusión.

MARCUSE, H. (2010). Razón y Revolución. Madrid: Alianza Editorial.

MARTÍNEZ, B. (2011). "Periodismo y responsabilidad social: el paradigma del tratamiento informativo de la violencia contra la mujer". En Revista de Comunicación Vivat Academia, ${ }^{\circ}$ 119, Madrid: Universidad Complutense de Madrid. p. 58-70.

MULVEY, K.; RICHARDS, M. (1998). Decades of Beauty: The Changing Image of Women 1890s 1990s. New York: Checkmark Books.

NASH, M. (2004). Mujeres en el mundo. Historia, retos y movimientos. Madrid: Alianza Editorial.

PADILLA, G. (2009a). "La mujer en el cine de Kenji Mizoguchi”. En: CIC Cuadernos de Información y Comunicación, $\mathrm{n}^{\circ}$ 14, Madrid: Universidad Complutense de Madrid. p. 251-267.

PADILLA, G. (2009b). "El éxito de Mujeres Desesperadas desde el Análisis Transaccional”. En: Revista de Análisis Transaccional y Psicología Humanista, nº 60, Madrid: AESPAT. p. 20-35.

PADILLA, G. (2010). "El mundo de la trilogía Millenium desde las perspectivas del Análisis Transaccional, la Ética, la Moral y la Política". En: Revista de Análisis Transaccional y Psicología Humanista, no 61, Madrid: AESPAT. p. 173-188.

PERRE, J. (1962). Les mutations de la guerre moderne. París: Payot.

PICTET. J. (1986). Desarrollo y Principios del Derecho Internacional Humanitario. Ginebra: Instituto Henry Dunant.

RADL PHILIPP, R.; GARCIA NEGRO M ${ }^{\mathrm{a}}$ C. (ed.) (1993). A Muller e a súa Imaxe. Santiago de Compostela: Universidad de Santiago de Compostela.

RENOUVIN, P. (1948). La crise européenne et la Première Guerre Mondiale. París: Presses Universitaires de France.

RENOUVIN, P. (1972). La Première Guerre Mondiale. París: Presses Universitaires de France.

RODRÍGUEZ, J. (2010). "De las programaciones didácticas a la unidad didáctica: incorporación de competencias básicas y la concreción de tareas". En: Revista Docencia e Investigación, $\mathrm{n}^{\circ}$ 9, Toledo: Universidad de Castilla La Mancha. p. 245-270. 
SCOTT, J. W. (1992). "El problema de la invisibilidad". En RAMOS ESCANDÓN, C. (Comp.) (1992). Género e historia. La historiografía sobre la mujer. México D.F.: Instituto Mora/UAM. p. 38-48.

SOTELO, J. (2012). "Deporte y social media: el caso de la Primera División del fútbol español". En: Historia y Comunicación Social, nº 17, Madrid: Universidad Complutense de Madrid. p. 217-230.

SULLEROT, E. (1968). Histoire et sociologie du travail féminin. París: Gonthier. VIDAURRETA, M. (1981). La guerra y la condición femenina en la sociedad industrial: el caso de Francia. Madrid: Ministerio de Cultura.

\section{Los autores}

Graciela Padilla Castillo es Profesora titular interina de la Facultad de Ciencias de la Información (Universidad Complutense de Madrid). Sus líneas de investigación abordan Estudios de Género, Ética audiovisual, Teoría de la Información, Ficción televisiva, Redes sociales y Redes profesionales, y Análisis Transaccional. Doctora en Ciencias de la Información, con Premio Extraordinario de Doctorado, ha completado su formación posdoctoral en la Universidad de California en Los Ángeles (UCLA). Es autora y coautora de más de cincuenta capítulos de libro y artículos publicados en revistas científicas. Miembro del Instituto de Investigaciones Feministas (Universidad Complutense de Madrid) y coordinadora del Máster universitario en Comunicación Política y Empresarial (Universidad Camilo José Cela).

Javier Rodríguez Torres es Doctor en Pedagogía, Máster en Psicología Escolar, Licenciado en Pedagogía y Profesor de EGB. Durante más de veinte años, ha trabajado en la Enseñanza no universitaria. Desde el curso 2004, es Profesor en la Universidad de la UCLM en el Departamento de Pedagogía. En la actualidad, es Secretario del Departamento. Profesor de distintos cursos de Postgrado de la UCLM. Miembro de distintos Proyectos de Investigación con las Universidades de Alcalá, UCLM, UNED, Poitiers (Francia) y UBA. Sus líneas de investigación abordan el currículum en torno a las diferencias de género y la inclusión de las TIC, los discursos de poder -saber que se generan en el uso y aplicación de las tecnologías, educación en valores y convivencia y educación para salud. Ha obtenido premios nacionales, autonómicos y provinciales a distintos proyectos de investigación e innovación educativa. 